\title{
Molecular characterization of two isoforms of defensin from hemocytes of the oyster Crassostrea gigas
}

\author{
M. Gonzalez ${ }^{1, a}$, Y. Gueguen ${ }^{\mathrm{a}}, \mathrm{G}$. Desserre ${ }^{\mathrm{a}}$, J. de Lorgeril $^{\mathrm{a}}$, \\ $B$. Romestand $d^{a}$ and $E$. Bachère ${ }^{a,{ }^{*}}$
}

aUMR5171, Génome Population Interactions Adaptation, IFREMER-CNRS-UMII, Université de Montpellier II, 2 Place Eugène Bataillon, CC80, 34095 Montpellier Cedex 5, France

*: Corresponding author : evelyne.bachere@ifremer.fr

\begin{abstract}
:
Antimicrobial peptides (AMPs) are important components of the host innate immune response against microbial invasion. We previously characterized the first AMP from an oyster, a defensin, that was shown to be continuously expressed in the mantle of Crassostrea gigas. In this study, we report the cDNA cloning of two new isoforms of the defensin AMP family (Cg-defh1 and Cg-defh2) from the hemocytes of the oyster. The deduced amino acid sequences reveal two peptides of 73 amino acid residues with a mature portion consisting of 43 amino acid residues. Cg-Defh1 and Cg-Defh2 share $86 \%$ amino acid identity and belong to the "arthropod-molluscs defensin family". qRT-PCR analyses indicate that $\mathrm{Cg}$-defh2 is continuously expressed in the hemocytes of $\mathrm{C}$. gigas. In addition, after a bacterial challenge, the level of Cg-defh2 transcripts decreases dramatically in the circulating hemocyte population and this decrease can be correlated with an increase of Cg-defh2 transcripts in the gill and the mantle tissue, suggesting a possible migration of the hemocytes expressing $\mathrm{Cg}$-defh2 towards the tissues implicated in the first defense barrier of the oyster. These results would suggest an important role of $\mathrm{Cg}$-Defh2 in the oyster response to a microbial challenge.
\end{abstract}

Keywords: Mollusc bivalve; Marine invertebrate; Pacific oyster; Hemocytes; Antimicrobial peptide; Defensin 


\section{Introduction}

It is widely known that antimicrobial peptides (AMPs) are major actors in innate immunity, conserved in evolution and present in all phyla of the living kingdom. These effectors present a great diversity in terms of structural features, biological properties and functions, and also in their tissue distribution and expression. [1, 2]. Defensins are compact cationic peptides, approximately 3-5 kDa in size, containing three or four disulphide bridges, and are active against a wide range of bacteria and fungi [3]. The vertebrate defensins can be grouped into three subfamilies, the $\alpha$-defensins and $\beta$-defensins, which are distinguished on the basis of the connectivity of their six cysteine residues, and the cyclic $\theta$-defensins [4]. In contrast with the classification of the vertebrate defensins based on their secondary structure, the grouping in clear distinct subfamilies of the invertebrate defensins is based on their biological properties, antibacterial versus antifungal [2]. Defensins are the most widespread family of invertebrate AMPs and more than 70 different defensins have been isolated in arthropods (insects, ticks, spiders, scorpions) and in molluscs [2]. In addition, the invertebrate defensins differ from the vertebrate defensins by their disulfide bridging [5].

To date, in molluscs, AMPs were only reported in the gastropod, Dolabella auricularia [6] and in the bivalves, the mussels Mytilus edulis [7] and M. galloprovincialis [8]. Based on a biochemical approach and molecular cloning, three groups of cationic cysteine-rich AMPs have been isolated from the hemocytes of unchallenged mussels, M. galloprovincialis: (i) defensinlike peptides, MGD-1 and MGD-2 [9]; (ii) myticins; and (iii) mytilins that have also eight cysteine residues but different specific cysteine arrays and amino acid sequences [10]. In the mussel, AMPs are only produced in hemocytes where they are stored and released following bacterial challenges [9]. Recently, two defensins from the oyster Crassostrea virginica [11] and Crassostrea gigas [12] were simultaneously discovered by two different groups. C. virginica defensin was purified from gill extract and contains 6 cysteines [11] whereas the defensin $\mathrm{Cg}$ Def, from C. gigas, was isolated from mantle tissue using Expressed Sequence Tag approach and contains 8 cysteines [12]. These two defensins display sequence homology with members of the "arthropod-molluscs defensins family". In our group, the C. gigas defensin from mantle has been fully characterized [12]. Cg-Def recombinant peptide was produced in Escherichia coli and displayed antibacterial activity. We observed that $\mathrm{Cg}$-def gene is continuously expressed in the mantle. Additionally, the "CS $\alpha \beta$ type" 3D structure of $C g$-Def was determined in aqueous solution by ${ }^{1} \mathrm{H}$ NMR spectroscopy and molecular modeling. In the present study, we report the cDNA cloning of two novel isoforms of defensin from C. gigas hemocytes and their gene expression analyses after bacterial challenge.

\section{Materials and Methods}

\section{Animals, tissues collection and immune challenge}

Adult oysters, Crassostrea gigas (3 years old; $12 \mathrm{~cm}$ ), were purchased from a local oyster farm in Palavas (Gulf of Lion, France) and kept in sea water at $15^{\circ} \mathrm{C}$. Oysters were stimulated by balneation with $5 \times 10^{8}$ bacteria/litre. The micro-organisms used for the challenge were the heatkilled bacterial strains Micrococcus luteus, Vibrio splendidus and Vibrio anguillarum. Hemolymph was collected at different times (24 and $48 \mathrm{~h}$ ) from the pericardial cavity through the adductor muscle under an equal volume of anti-aggregant Modified Alsever Solution (27 $\mathrm{mM}$ Na-citrate, $336 \mathrm{mM} \mathrm{NaCl}, 115 \mathrm{mM}$ glucose, $9 \mathrm{mM}$ EDTA, pH 7.0) and immediately centrifuged at $700 \mathrm{~g}$ for 10 minutes $\left(4^{\circ} \mathrm{C}\right)$ to separate the blood cells from plasma. After hemolymph collection, oyster tissues were harvested by dissection. Hemocytes and tissues (gills and mantle) were further treated according to different experimental procedures (see below). The experiments were done in triplicate and, to minimize individual variability, at least ten oysters were used in each experimental condition 


\section{RT-PCR and molecular cloning}

Total RNA was extracted from the hemocytes of non stimulated C. gigas using Trizol reagent according to manufacturer's instructions (Invitrogen ${ }^{\mathrm{TM}}$ ) and treated with DNAse Turbo (Ambion). Following heat denaturation $\left(70^{\circ} \mathrm{C}\right.$ for $\left.5 \mathrm{~min}\right)$, reverse transcriptions was performed using $1 \mu \mathrm{g}$ of total RNA prepared with $50 \mathrm{ng} / \mu \mathrm{l}$ oligo- $(\mathrm{dT})_{12-18}$ in a $50 \mu \mathrm{l}$ reaction volume containing $1 \mathrm{mM}$ dNTPs, 1 unit/ $\mu$ l of RnaseOUT (Invitrogen ${ }^{\mathrm{TM}}$ ) and 200 units/ $\mu \mathrm{l}$ M-MLV reverse transcriptase in reverse transcriptase buffer. The cDNAs were amplified using primers Deffw 5' TTGCTGAGTAGAATGAAAGTATTCG 3' and Defrev, 5' CAGATTGCATAAAGATTTATTACACG 3' designed in the untranslated region of the cDNA sequence of $\mathrm{Cg}$-Def (Genbank accession number CAJ19280) [12]. The amplification program consisted of $3 \mathrm{~min}$ at $95^{\circ} \mathrm{C}$, followed by 34 cycles of $95^{\circ} \mathrm{C}$ for $30 \mathrm{~s}, 55^{\circ} \mathrm{C}$ for $30 \mathrm{~s}, 72^{\circ} \mathrm{C}$ for $30 \mathrm{~s}$ min and a final elongation step of $72^{\circ} \mathrm{C}$ for $3 \mathrm{~min}$. Amplified products were analyzed on $1,5 \%$ agarose gels, cloned into pCR 2.1 TOPO TA cloning vector (Invitrogen ${ }^{\mathrm{TM}}$ ) and sequenced from both directions with $\mathrm{T} 7$ and $\mathrm{T} 3$ primers. The nucleotide sequences reported in this paper has been submitted to the GenBankTM/EBI Data Bank with accession numbers DQ400101 and DQ400102.

\section{Sequence analysis}

General homology searches were performed with the BLAST software on the NCBI home page (http://www.ncbi.gov./Blast). Deduced amino acid sequences were aligned by ClustalX (http://www.ch.embnet.org/software/ClustalW.html). Phylogenetic and molecular evolutionary analyses were conducted using MEGA version 3.0. The tree was built by the Minimum Evolution method based on the alignment of the sequences using ClustalX (alignment was improved using the Seaview software). Bootstrap values (\%) of 1000 replicates were calculated for each node of the consensus tree obtained.

\section{Real Time PCR analyses}

Real-time polymerase chain reaction (rtPCR) analysis was done to determine whether acute changes in Cg-defh1 and $C g$-defh2 RNA abundance could be detected from hemocytes, gills and mantle tissues, sampled 24 and 48 hours post-stimulation. Pooled samples tissues total RNA (10 individuals per pool) were analysed from non stimulated and stimulated oysters at 24 and 48 hours post-challenge. For Cg-defh1, the forward and reverse primers were Defh1F (5'AGTATTCGGACTTTTTACATTGGT-3') and Defh1R (5'CCGCTCTACAACCGATGGATTG-3'). For $\mathrm{Cg}$-defh2, the forward and reverse primers were Defh2F (5'-GTATTCGTACTTCTTACATTAGC-3') and Defh2R (5'GCTCTACAACCGATGGACCT-3'). The gene encoding the elongation factor (e.f.) was used as internal control. For e.f., the forward and reverse primers were, EF (5'ATGCACCAAGGCTGCACAGAAAG-3') and EFR (5'-TCCGACGTATTTCTTTGCGATGT3’), respectively.

Total RNAs were treated with DNase (TURBO DNase, Ambion) to eliminate contaminating genomic DNA. The Dnase was removed by phenol chloroform extraction. First strand cDNA was synthesized using polydT primers (Oligo(dT)12-18, Invitrogen). Reverse transcription was performed on $1 \mu \mathrm{g}$ total RNA using the SUPERScript ${ }^{\mathrm{TM}} \mathrm{RNase}^{-}$reverse transcriptase (Invitrogen) according to the manufacturer's instructions. PCR amplifications were realized with the LightCycler ${ }^{\mathrm{TM}}$ (Roche) in the presence of SYBR-Green ${ }^{\mathrm{TM}}$ (Master SYBR Green $\left.^{\mathrm{TM}}\right)$. Briefly, the following components were mixed to the indicated end-concentration: 5 $\mathrm{mM} \mathrm{MgCl}_{2}, 0.5 \mu \mathrm{M}$ of each primer, $1 \mu \mathrm{l}$ of reaction mix (LC Fast Start Master SYBR Green I; Roche Diagnostics) in a final volume of $9.5 \mu$ l. Reverse transcribed RNA (0.5 $\mu$ l) was added, as PCR template, to the LightCycler master mix and the following run protocol used: initial 
denaturation at $95^{\circ} \mathrm{C}$ for $10 \mathrm{~min} ; 95^{\circ} \mathrm{C}$ for $15 \mathrm{~s} ; 62^{\circ} \mathrm{C}$ for $15 \mathrm{~s} ; 72^{\circ} \mathrm{C}$ for $15 \mathrm{~s}$ with a single fluorescence measurement; melting curve program $\left(73-95^{\circ} \mathrm{C}\right.$ with a heating rate of $0.1^{\circ} \mathrm{C}$ per second and a continuous fluorescence measurement) and finally a cooling step to $30^{\circ} \mathrm{C}$. For further expression level analysis, the crossing points (CP) were determined for each transcript using the LightCycler software. Specificity of RT-PCR product was analyzed on agarose gel and melting curve analysis. The copy ratio of each analyzed cDNA was determined as the mean of three replicates.

The relative expression ratio of and $C g$-defh1 was calculated based on the CP deviation of each RT-PCR product of RNA extracted from stimulated oyster versus the appropriate control sample (RT-PCR product of RNA from oyster non stimulated), and expressed in comparison to the reference gene (elongation factor, e.f.). The relative expression ratio of $\mathrm{Cg}$ - $d e f$ was calculated based on the delta-delta method for comparing relative expression results defined as: ratio $=2^{-}$ $\left[\Delta\right.$ CPsample $-\Delta$ CPcontrol] $=2^{-\Delta \Delta \mathrm{CP}}[13]$.

In order to determine the expression pattern of $C g$-defh 1 and $C g$-defh2 during bacterial challenge, two batches of oysters were prepared. In the first one, oysters were stimulated by bath with killed bacteria and in the second one, non-stimulated oysters ("naïve oyster") were used as control. Then, qRT-PCR analyses were performed, with total RNA extract from hemocytes, mantle and gill, collected at two time points post-challenge (24 and $48 \mathrm{~h}$ ).

\section{Results and discussion}

\section{Characterization of two defensin cDNA from C. gigas hemocytes}

Invertebrates rely solely on innate immune mechanisms that included both humoral and cellular response. Humoral immunity in marine invertebrates is characterized by antimicrobial agents present in the blood cells and plasma [14]. The involvement of AMPs in natural resistance to infection is sustained by their strategic location in phagocytes, in body fluids and at the epithelial level. We previously identified and characterized the first antimicrobial from the oyster C. gigas. $\mathrm{Cg}$-Def defensin was isolated from $C$. gigas mantle tissue and was shown to be exclusively and continuously expressed in this tissue, suggesting a key role of this peptide in the antimicrobial defenses of the oyster by providing a first line of defense against colonization by pathogens [12]. In the present study, a PCR strategy was used to identify and clone two defensin isoforms from C. gigas hemocytes. PCR primers were designed from the 5' and 3'-untranslated regions of Cg-def. A single product of around 300bp was amplified by PCR. After cloning and sequencing, we obtained two different deduced amino acid sequences with $86 \%$ of identity and showing evident homology with the defensin AMP family. The full length nucleotide and aminoacid sequences of the two new isolated defensin isoforms from $C$. gigas hemocytes $(\mathrm{Cg}$-Defh1 and $C g$-Defh2) are presented in Figure 1. The two cDNA sequences present a 195 bp region encoding a 65 amino acid peptide. The deduced amino acid sequence starts with a signal peptide and the cleavage site for signal peptidase is most likely located after the alanine residue preceding the glycine in position 23, as predicted by SignalP 3.0 software (data not shown).

The amino acid sequences of the mature peptides, $C g$-Defh1 and $C g$-Defh2, were aligned with defensins from the "arthropod defensin family" available in GenBank, that contains defensins from arthropods and molluscs (Figure 2A). The position of the cysteines is well conserved, however, as observed with the defensins MGD-1 and MGD-2 from $M$. galloprovincialis hemocytes and $C g$-Def from C. gigas mantle, $C g$-Defh1 and $C g$-Defh2 have four disulfide bonds. This additional bridge was proposed to render the peptide more stable in high osmolarity media such as in sea water [15]. In addition, it is noteworthy that, Seo et al., have recently isolated a defensin from the eastern oyster $C$. virginica and the corresponding 4265,0 Da peptide has 38 amino acids with only six cysteines [11]. Cg-Defh1 and Cg-Defh2 share 84 and 79\% identity, respectively, with the mantle $C g$-Def and both peptides show 51\% identity with the $C$. virginica defensin. Less identity was observed with defensins from the 
scorpion Androctonus australis, the dragonfly Aeschna cyanea and the tick Dermacentor variabilis (Figure 2A).

Phylogenetic tree was constructed with $C g$-Defh1 and $C g$-Defh2 amino acid sequences and the corresponding sequences from members of the invertebrate defensin family. The tree showed that the sequences were split into three clearly distinct groups (Figure 2B). The oyster defensins clustered together with mussel defensins and split up with the second group that comprises paleopteran insect (dragonfly) and arachnids (scorpion and tick) defensins. In addition, all defensins from neopteran insects clustered in the third group (Figure 2B) [16]. It is noteworthy that plectasin, the first fungal defensin described; shows a high degree of structural and sequence similarity to the defensins of invertebrates [17]. The similarity of plectasin to invertebrate defensins adds weight to a possible common genetic origin for the defensins of plants, fungi and protostome invertebrates. However, confirmation of this hypothesis will require the characterization of additional defensins from other fungi and/or invertebrates [17].

\section{Real-Time PCR Analyses of Cg-def transcript levels after oyster bacterial challenges}

qRT-PCR analyses were performed to determine the expression pattern of $C g$-defh1 and Cg-defh2 in hemocytes, mantle and gill during bacterial challenge. qPCR analysis revealed that the amount of $\mathrm{Cg}$-defh1 transcript measured during the experiment was very low; then, it was difficult to measure acute expression profile pattern of $\mathrm{Cg}$-defh1 (data not shown). Concerning, Cg-defh2, qPCR analysis indicates that, in "naïve-oysters", this defensin form is expressed continuously. However, significant decrease of $\mathrm{Cg}$-defh2 transcripts was evidenced in circulating hemocytes after a bacterial challenge. Indeed, very low level of $\mathrm{Cg}$-defh2 transcripts were detected 24 and 48 hours post bacterial challenge (Figure 3). Such results could explain why, in a previous EST project performed with RNA from hemocytes of oysters collected $12 \mathrm{~h}$ postbacterial challenge, we did not isolate any EST for defensins [18].

In addition, further investigation of the effects of the challenge on the tissue distribution of $\mathrm{Cg}$-defh2 revealed an increase of the level of $\mathrm{Cg}$-defh2 transcripts in the oyster mantle and gill, at 24 and 48 hours post-challenge (Figure 3). Because as for all experiments, the temperature melting curve and the size of the qPCR products were identical, we can assume that, we detected in the mantle and the gill tissues, transcripts of the same defensin expressed from hemocytes. These results would suggest that, after a challenge, the hemocyte population that expresses $\mathrm{Cg}$-defh2 could migrate towards the tissues implicated as a first defense barrier of the oyster. This pattern of expression is the same as the one observed for the defensin MGD- 1 in the mussel M. galloprovincialis after a bacterial injection where hemocytes have been shown to concentrate at the site of injection [9]. Our data also corroborate the results obtained with the $C$. virginica defensin [11]. Indeed, this peptide was originally purified from the gills of the oyster but the authors proposed that they might originate from circulating hemocytes that are abundant in gill filaments [11]. Thus, it appears that in molluscs, defensin genes are not regulated as in insects, where the expression of the genes encoding antibacterial peptides is induced after bacterial challenge [19]. Consequently, the decrease of messenger concentration observed from the hemocytes could be due to an under-representative of the $C g$-defh2 producing cells in the circulation. Further studies investigating the ratio of the cells expressing $C g$-defh2, after challenge, in the total population of circulating hemocytes are necessary to confirm this hypothesis.

\section{Conclusion}

The data in this report, which represent the first characterization of an antibacterial peptide from the hemocytes of an oyster, indicate a close relationship between the oyster defensins from hemocytes (Cg-Defh1, $C g$-Defh2) and mantle (Cg-Def). Additional investigations of oyster immune peptides and their expression are underway to better understand 
the role of these AMPs in the oyster immune response. However, preliminary results would suggest that the different isoforms of the defensins, $C g$-Defh1, $C g$-Defh2 from hemocytes and $\mathrm{Cg}$-Def from mantle could be detected in one individual. Further studies on the influence of microbial challenge on peptide production and localisation, together with a determination of their complete activity spectra will permit us to elucidate the reason for such diversity.

\section{Acknowledgments}

This study was part of a collaborative project supported by the European Commission, DG XII, in the program International Co-operation with Developing Countries, INCO-DC, contract ICA4-CT-2001-10023 (IMMUNAQUA). Work in the authors' laboratory was supported in part by the Ifremer, the CNRS and the University of Montpellier II. This study was also part of a French program MOREST funded by Ifremer, the Regions Basse-Normandie, Bretagne, Pays de la Loire and Poitou-Charentes and by the Conseil Général du Calvados. M. Gonzalez was supported by a doctoral funding from Programme CONICYT-BID. We are very grateful to E. Desmarais and P. Bulet for helpful discussion and M. Leroy for technical assistance.

\section{References}

[1] Bachère E, Gueguen Y, Gonzalez M, de Lorgeril J, Garnier J, Romestand B. Insights into the antimicrobial defense of marine invertebrates: the penaeid shrimps and the oyster Crassostrea gigas. Immunol Rev 2004;198:149-168.

[2] Bulet P, Stocklin R, Menin L. Anti-microbial peptides: from invertebrates to vertebrates. Immunol Rev 2004;198:169-84.

[3] Boulanger N, Lowenberger C, Volf P, Ursic R, Sigutova L, Sabatier L, et al. Characterization of a defensin from the sand fly Phlebotomus duboscqi induced by challenge with bacteria or the protozoan parasite Leishmania major. Infect Immun 2004;72:7140-6.

[4] Harder J, Bartels J, Christophers E, Schroder JM. Isolation and characterization of human beta -defensin-3, a novel human inducible peptide antibiotic. J Biol Chem 2001;276:5707-13.

[5] Dimarcq J-L, Bulet P, Hétru C, Hoffmann JA. Cysteine-rich antimicrobial peptides in invertebrates. Biopolymers 1998; 47:465-477.

[6] Iijima R, Kisugi J, Yamazaki M. A novel antimicrobial peptide from the sea hare Dolabella auricularia. Dev Comp Immunol 2003;27:305-11.

[7] Charlet M, Chernysh S, Philippe H, Hétru C, Hoffmann JA, Bulet P. Isolation of several cysteine-rich antimicrobial peptides from the blood of a mollusc, Mytilus edulis. J. Biol. Chem 1996; 271:21808-21813.

[8] Hubert F, Noël T, Roch P. A member of the arthropod defensin family from the edible Mediterranean mussels (Mytilus galloprovincialis). Eur J Biochem 1996;240:302-306.

[9] Mitta G, Vandenbulcke F, Hubert F, Roch P. Mussel defensins are synthesised and processed in granulocytes then released into the plasma after bacterial challenge. J Cell Sc 1999;112:4233-4242.

[10] Mitta G, Vandenbulcke F, Hubert F, Salzet M, Roch P. Involvement of mytilins in mussel antimicrobial defense. J Biol Chem 2000; 275:12954-12962.

[11] Seo JK, Crawford JM, Stone KL, Noga EJ. Purification of a novel arthropod defensin from the American oyster, Crassostrea virginica. Biochem Biophys Res Commun 2005; 338:1998-2004. 
[12] Gueguen Y, Herpin A, Aumelas A, Garnier J, Fievet J, Escoubas JM, et al. Characterization of a defensin from the oyster Crassostrea gigas. Recombinant production, folding, solution structure, antimicrobial activities, and gene expression. J Biol Chem 2006; 281:313-23.

[13] Livak, K.J. and T.D. Schmittgen, Analysis of relative gene expression data using realtime quantitative PCR and the 2(-Delta Delta C(T)) Method. Methods 2001, 25:402-408.

[14] Tincu JA, Taylor SW. Antimicrobial peptides from marine invertebrates. Antimicrob Agents Chemother 2004;48:3645-54.

[15] Yang YS, Mitta G, Chavanieu A, Calas B, Sanchez JF, Roch P et al. Solution Structure and Activity of the Synthetic Four-Disulfide Bond Mediterranean Mussel Defensin (MGD - 1). Biochemistry 2000;39:14436-14447.

[16] Froy O, Gurevitz M. Arthropod and mollusk defensins evolution by exon-shuffling. Trends Genet 2003;19:684-687.

[17] Mygind PH, Fischer RL, Schnorr KM, Hansen MT, Sonksen CP, Ludvigsen S, et al. Plectasin is a peptide antibiotic with therapeutic potential from a saprophytic fungus. Nature 2005;437:975-80.

[18] Gueguen Y, Cadoret JP, Flament D, Barreau-Roumiguiere C, Girardot AL, Garnier J, et al. Immune gene discovery by expressed sequence tags generated from hemocytes of the bacteria-challenged oyster, Crassostrea gigas. Gene 2003;16:139-145.

[19] Hoffmann JA, Reichhart JM and Hetru C. Innate immunity in higher insects. Curr Opin Immunol 1996;8:8-13. 

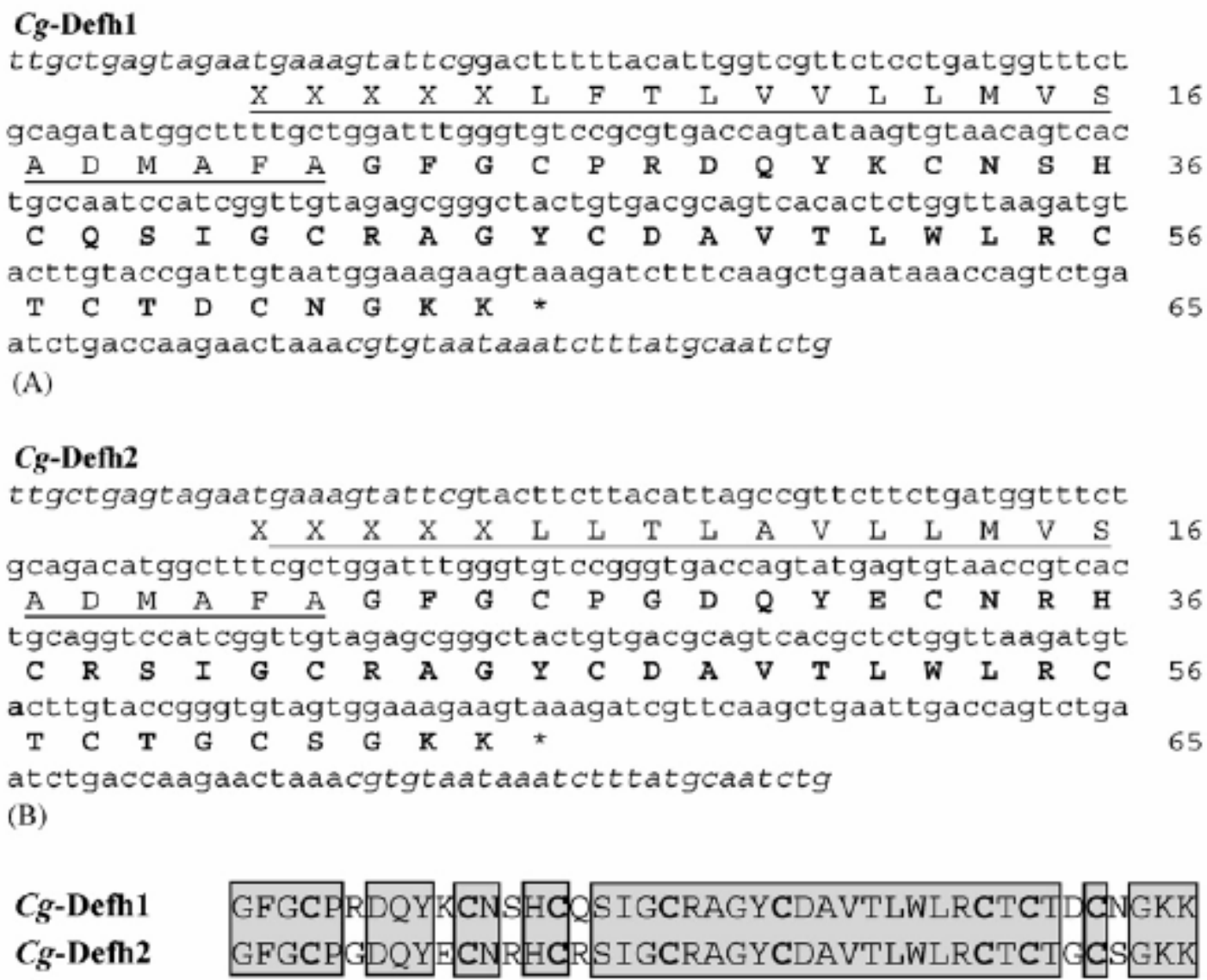

Fig. 1. Nucleotide and deduced amino acid sequences of Cg-Defh1 (A) and Cg-Defh2 (B) cDNAs and alignment of mature defensins (C). (A, B) The deduced amino acid sequences of the ORF are indicated above the nucleotide sequence. Asterisks indicate the stop codons. Amino acids are numbered on the right. The incomplete sequences of signal peptides are underlined and mature peptides are indicated in bold letters. The sequences of the primers used for Cg-defh1 and Cg-defh2 cDNA cloning are in italics. (C) Cysteines are in bold and identical residues are boxed. 


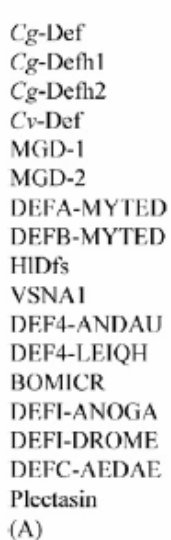

(A)
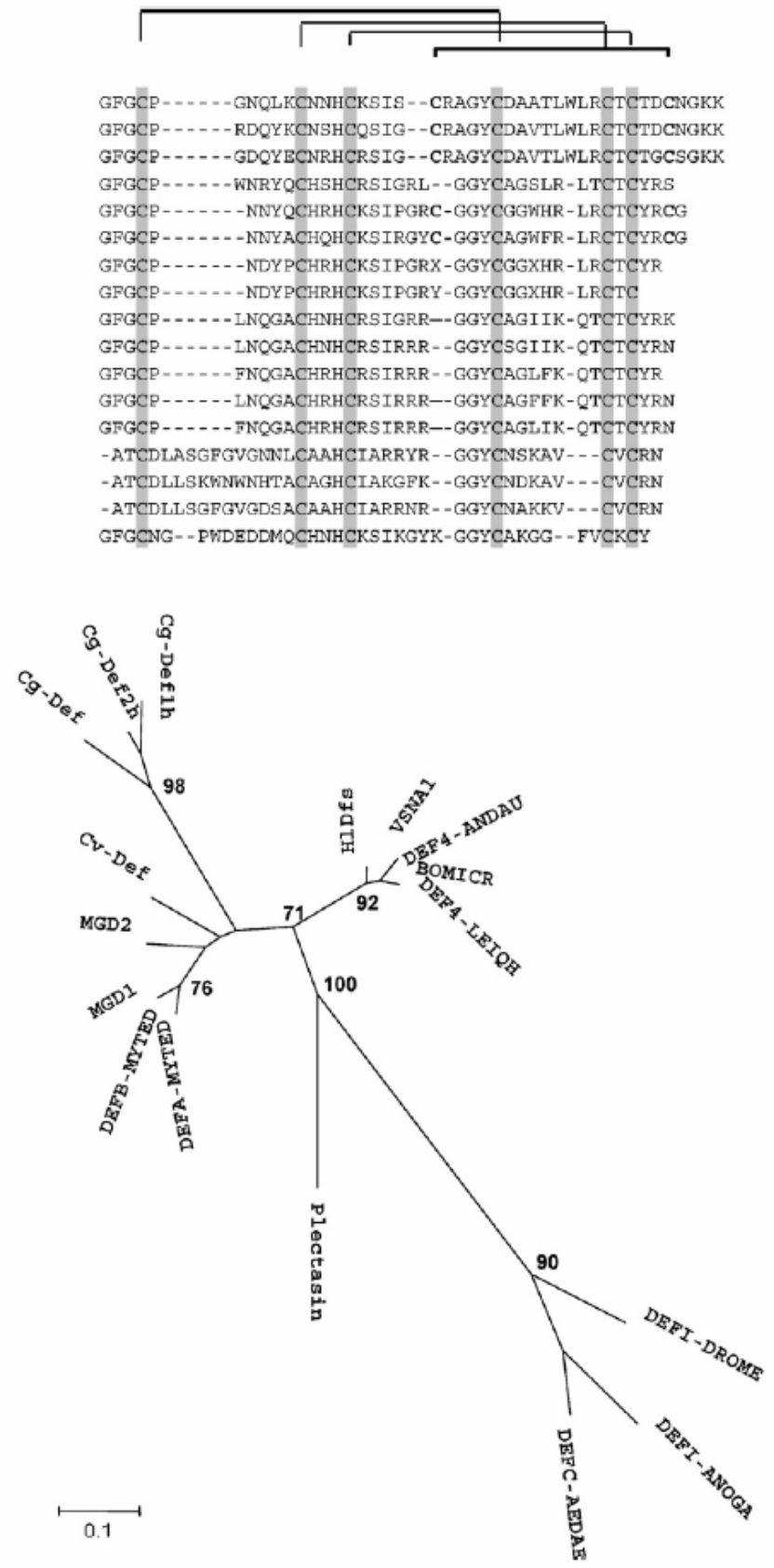

Figure 2. (A) Sequence alignment of defensins from arthropods and molluscs. Defensin obtained from hemocytes were alignment with others invertebrate defensins. Conserved cysteine residues are shaded. The two additional cysteine residues found in the $C g$-Def mantle, $C g$-Def A and B, MGD-1 and MGD-2 are in bold. The disulfide bonds are indicated. (B) Phylogenetic tree of invertebrate defensins. The tree was generated using an alignment made using CLUSTAL X. From this alignment a distance based phylogenetic tree was constructed using the Minimum Evolution method of the MEGA package. The bootstrap values of the branches are indicated. Oyster Crassostrea gigas (Cg-Def [AJ565499], Cg-Defh1 [DQ400101] and Cg-Defh2 [DQ400102]) and Crassostrea virginica (Cv-Def, [11]); mussels Mytilus edulis (DEFA-MYTED, [P81610], DEFB-MYTED, [P8161]) and M. galloprovincialis (MGD-1, [P80571]; MGD-2, [AAD52660]); tick Dermacentor variabilis (varisin, VSNA1, [AAO24323]), scorpion Androctonus australis (DEF4-ANDAU, [P56686]), the african malaria mosquito Anopheles gambiae (DEFI-ANOGA, [Q17027]), fruit fly Drososphila melanogaster (DEFIDROME, [P36192]) and yellowfever mosquito Aedes aegypti (DEFC-AEDAE, [P81603]). Haemaphysalis longicornis (HIDfs, [BAD93183]), Boophilus microplus (BOMICR, [AAO48943]), Leiurus quinquestriatus (DEF4-LEIQH, [P41965]) and the saprophytic fungus Pseudoplectania nigrella (plectasin, [CAI83768]. The accession numbers are in brackets. 


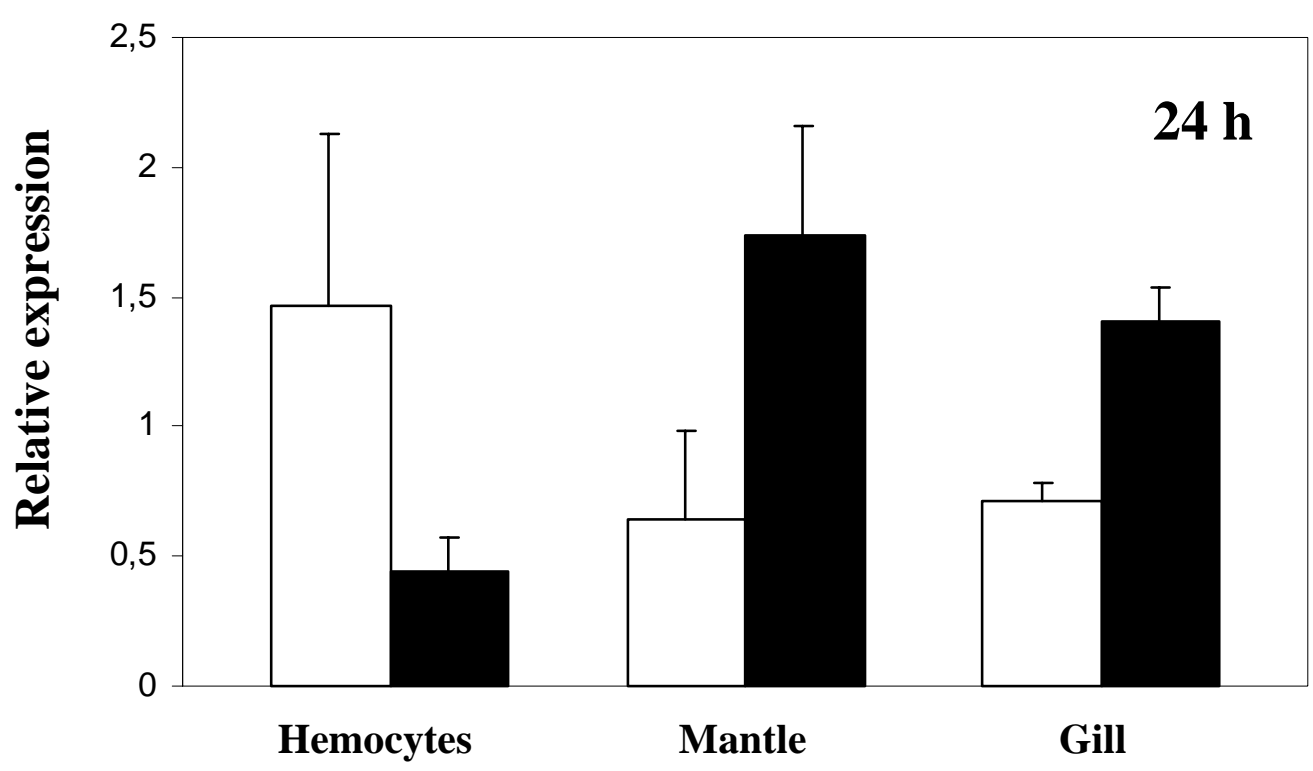

48h post-challenge

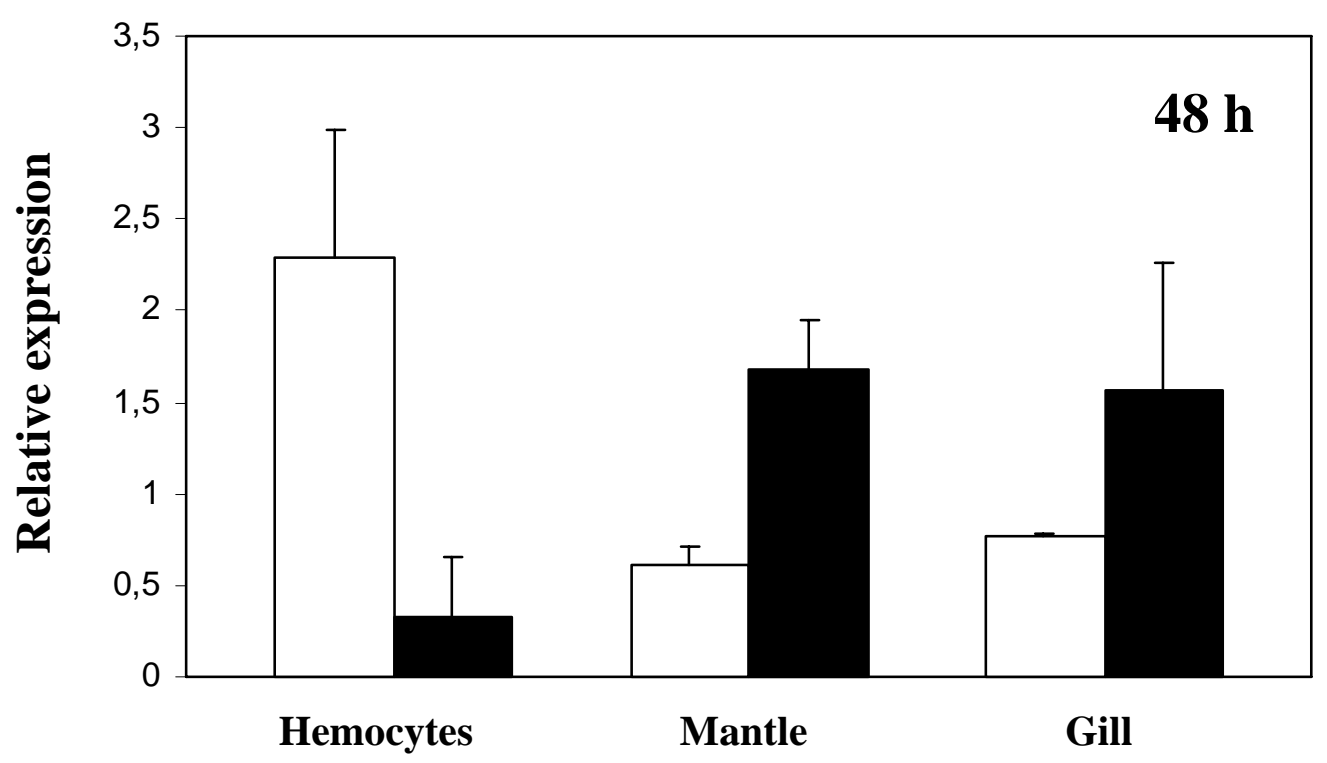

Figure 3. Expression of $\mathbf{C g}$-defh $\mathbf{2}$ mRNA analyzed by real time quantitative RT-PCR. $\mathrm{Cg}$-defh2 mRNA expression was measured in hemocytes, mantle and gills following bacterial challenge. Results are means \pm SE of three independent experiments realized on a pool of ten oysters from non-stimulated (white) and stimulated (black) oysters, at 24 and 48 h postinfection. Bars represent the relative $\mathrm{Cg}$-defh2 transcript levels normalized to elongation factor (e.f.) transcript levels, as described in Materials and Methods. The $\Delta \Delta \mathrm{CP}$ values for each experimental condition were obtained by subtraction of mean of all $\Delta \mathrm{CPs}$ (relative expression of the mean $=1$ ). 